\title{
Study on the Growth of the Fatigue Crack under Flexural Moment
}

\author{
Wen Zhong, Jiajie Hu, Jun Guo, Zibin Li \& Quyue Liu \\ State Key Laboratory of Traction Power, Sout hwest Jiaotong University, Chengdu 610031, China \\ E-mail:zw1019@126.com
}

The research is financed by the National Natural Science Fund of China (No.50675183) and the National Key Base $R \& D$ Plan of China (Plan 973, No.2007CB714702) and the Innovation Team Cultivation Plan of Southwest Jiaotong University (No.2007IRT01). (Sponsoring information)

\begin{abstract}
Vehicles will suffer various loads in the moving process, and under the functions of various factors such as the ovality of the wheel, and the impacts to the rails when the vehicle passes the curve rail, the rail slot and the turnoff, the wheels will inevitably add normal loads to the rails, and the flexural moment will influence the generating and growth of the fatigue crack. To study the influences of flexural moment on the fatigue performances of rails with various materials, the experiments about the growth performances of the fatigue cracks of two kinds of materials including U71Mn and PD3 under the flexural moment are made on the NENE-2 fretting test machine, and according to the experiment results, the measures to reduce the fatigue of rails are proposed. The research analysis and experiment results indicate that the fatigue crack generates from the focal point of stress at first, and the main shearing stress is very important, and when the static load functions, the direction of crack growth will change by a large angle, but when the dynamic load functions, the direction of crack growth will be stable, and under same loading speed, the fatigue crack of PD3 rail more easily generates and grows than U71Mn rail. To prevent and reduce the fatigue of rails, the selection of rail should accord with the type of the route and the actual working environment of the rail, and in the heavy freight route, the PD3 rail with high mechanical strength should be selected, and in the high-speed route, the U71Mn rail should be selected.
\end{abstract}

Keywords: Rail steel, Fatigue crack, Flexural moment, Growth, Research

With the increase of railway transportation and the enhancement of train speed, the destroys induced by the wheel track rolling contact fatigues become more and more seriously, especially in the high-speed routes and the heavy freight routes (Jin, 2001, P.92-108 \& Lu, 2007, P.41-54). For the mechanism of rolling contact fatigue, the prediction of crack life, the parameters with influencing functions and the methods of prevention, scholars have made large numbers of researches (Anders, 2005, P.1288-1300 \& Liu, 2000, P.30-34).

The twisting force between the wheels and rails can always produce plastic distortion of rails when the train moves (Liu, 1998, P.337-340). At the same time, the ovality will occur in wheels because of various reasons (such as machining and fretting), and the normal load will occur in movements and impact the rails. In addition, when the transverse motion and the yaw motion happens in the wheel pairs, especially when the vehicle passes the curves with small radius, the rail slot and the turnoff, the impact vibration will occur between wheel tracks. But because of the installing form of the rails, the normal load will make the rails to bend and distort adown, i.e. the under-area of the rail will be elongated by the pulling function, and the top-area of the rail will be reduced by the pulling function, and the energy dissipation will be different with the pure twisting force (Jonas, 2001, P.575-586). And if the fatigue crack is generated on the surface or the hypo-surface of the rails (A. Bernasconi, 2005, P.973-980), the rails will suffer the functions of reducing or pulling. Under the function of repetitive loading, the plastic distortion will be accumulated and form micro-cracks on the surface or the hypo-surface of the materials (Liu, 2002, P.1596-1599), i.e. the flexural moment will influence the generation and growth of the fatigue cracks.

On the NENE-2 fretting test machine, the growth characters of fatigue cracks of two kinds of rails including U71Mn and PD3 are studied under the flexural moment by improving the experiment clump, and the influences of the flexural moment on the generation and growth of rail fatigue crack are analyzed as follows. 


\section{Experiment}

\subsection{Experiment equipment}

The experiment is made on the NENE-2 fretting test machine, and the experiment equipment is seen in Figure 1. The whole experiment equipment is divided into the under-part and the top-part. The clump chuck and the loading chuck compose in the top-part which generates the normal load, and the simulated girder and the installing seat compose in the under-part of the equipment. To be like with the practical installing form of the rails, the bolt connection is adopted between the simulated girder and the installing seat, and two ends forms the fixed-end restriction. The installing seat is installed on the piston seat of the NENE-2 fretting test machine by the bolt, and it can realize the loading of normal load by the pumps of the pistons in the experiment.

In the experiment, the load extent is $20-350 \mathrm{~N}$ (U71Mn), or 20-350N, or 20-200N (PD3), and the loading speed is $12 \mathrm{~mm} / \mathrm{min}$.

To simulate the practical working condition, the maximum contact pressure in the experiment keeps equal with the maximum contact pressure of rails in the practical working condition (Liu, 2003, P.132-135). The size of the simulated girder (length $=220 \mathrm{~mm}$, breath $=8 \mathrm{~mm}$, height $=10 \mathrm{~mm}$ ) is confirmed by the working platform of the test machine. The crack of $0.18 \mathrm{~mm} \times 7.5 \mathrm{~mm}$ is prefabricated in the just middle of the simulated girder by the line cutting method.

Approximate the contact between the loading chuck and the simulated girder as the contact of two columns, and according to Hertz's contact theory,

$$
R=\left(\frac{1}{R_{1}}+\frac{1}{R_{2}}\right)^{-1}
$$

Because the radius of the girder $\mathrm{R}_{2}$ is close to $\infty$, so the radius of the loading chuck is

$$
R=\frac{P E^{*}}{\pi P_{0}^{2}}
$$

Where,

$$
E^{*}=\left(\frac{1-v_{1}^{2}}{E_{1}}+\frac{1-v_{2}^{2}}{E_{2}}\right)^{-1} .
$$

Take $\mathrm{P}=55 \mathrm{~N} / \mathrm{mm}, \mathrm{P}_{0}=1.2 \mathrm{Gpa}$ and $\mathrm{E}=2.14 \times 10^{5} \mathrm{Mpa}$ to compute, and consider the influences of the machining precision, take the radius of the loading chuck is $\mathrm{R}=1.3 \mathrm{~mm}$.

\subsection{Experiment materials}

Take the hot rolled rails of U71Mn and PD3 from the middle of the sample rails, and their chemical components and mechanical performances are seen in Table 1 and Table 2 (Zhao, 2007).

To compare the growths of cracks, the static loading experiment is implemented. When the static load functions, the clump chuck is fixed on the bracket, and the installing seat is put on the top-pole of the hydraulic pressure jack. By moving the top-pole of the jack continually until the sample ruptures, the static load can be implemented. After the experiment, take the prefab crack part in the middle of the simulated girder, and metallographically burnish and polish it, and observe the growth conformation of the crack by the optical microscope.

\section{Results and discussion}

In Figure 3 and Figure 4, the crack in the static loading will turn to the large-angle of $90^{\circ}$ when it growth to certain extent, and then grow randomly to other side. But in Figure 5 and Figure 6, the growth direction of fatigue crack is stable and changes little.

That is because when the static load functions, the energy can only rapidly release by the growth of the crack. And once the crack growth encounters the disfigurement in the interior of the material, it will change the direction at once to the direction that the edge stress of disfigurements centralizes. But because the crack will always grow to the direction that the energy consumes little, so when the direction grows to a little distance, it will return to the initial growth direction. But in the growth process of fatigue growth, the plastic distortion near the crack will consume part of energies, and make the release of energy to be slow, so the crack will grow always along the direction that the energy consumes least, and the direction will change little.

In Figure 3, Figure 4, Figure 5 and Figure 6, the generating points of the fatigue crack are located in the side of the top end of the crack round arc, not the middle of the round arc. From the analysis of the stress distribution, because the contact width is narrow, the pressure of the loading chuck can be simplified as the even distribution. According to

Hertz's contact theory, ignore the influences of prefab cracks, the value of the main shearing stress is $\tau_{1}=\frac{p}{\pi} \sin \alpha$, and its distribution is seen in Figure 7. 
In the ideal situation of Figure 7, from the main shearing stress distribution, the point of intersection of the isoline of the main shearing stress and the round arc of the top of the prefab crack is on the round arc of the side of the middle line (the macula in the Figure), and the crack is generates at this position. The maximum point of the main shearing stress is close to the generating point of the crack, which indicates that the main shearing stress significantly influence the generating position of the crack.

By the software of ANSYS, the finite element method (FEM) is used to further analyze the stress of the simulated girder. First, establish the solid model of the simulated girder of $220 \times 10 \times 8$, and generate the prefab crack of $7.5 \times 0.18$ by the Boolean subtraction calculating. Because the contact pressure is only related with the geometric forms of the load and contact interface, so the loading chuck can be simplified as a half column when the radius of the loading end doesn't change. Considering that the forms and the contact of the simulated girder and the loading chuck are simple, the gridding division adopts the intelligent mode of ANSYS. The two ends of simulated girder and the faces of X, Y and Z between the simulated girders are not displaced, and the load is implemented on the top of the loading end, and the load is $300 \mathrm{~N}$ when considering the practical experiment. The friction coefficients of the round arc face of the loading chuck and the surface of the simulated girder are 0.3 , and the stress distribution of the prefab crack top under the $300 \mathrm{~N}$ normal load is seen in Figure 8 by the solving and processing of the integrator in the software. And from the Figure, the generating position and the stress centralized position of the cracks good feature of superposition.

In most situations, the crack lengths of two kinds of rails will grow with the increase of the cycle times. At the initial stage, the crack grows little, and with the time goes, the growth speed of crack increases rapidly and goes into the fatigue rupture stage, and under the loading function, the example component will be broken (Wang, 2007, P.1026-1029). By the optical microscope, the load extent of the hot rolled rail of U71Mn is $20-350 \mathrm{~N}$, and the under the working situation of the loading speed of $12 \mathrm{~mm} / \mathrm{min}$, the obvious cracks will occur after 12000 times cycle (seen in Figure 5). After 21000 times cycle, the length of crack doesn't increase obviously with the former. The hot rolled rail steel of PD3 produces static loading crack when the loading extent is $20-350 \mathrm{~N}$. When the loading extent is $20-200 \mathrm{~N}$ and the loading speed is $12 \mathrm{~mm} / \mathrm{min}$, the obvious fatigue crack will occur after 4000 times, which indicates that under same loading speed and flexural function, the U71Mn has better anti-fatigue crack growth performance than PD3.

\section{Conclusions}

(1) The fatigue cracks generates from the focus point of the stress, and the focus point is the important factor to generate cracks, and the main shearing stress occupies important function, so the crack generates in the static loading is significantly different with the growth conformation of the fatigue crack.

(2) When the loading speeds are same, under the flexural moment produced by normal load, the fatigue crack of PD3 more easily generates and grows than U71Mn.

(3) Above analysis shows that two kinds of rail steel including PD3 and U71Mn presents different performances. With increase of railway transportation and speed, the working condition of rails will change largely. To prevent and reduce the fatigues of rails, the rail steel should be selected according to the practical working environment of rails. For example, in the heavy freight rout, the fretting is the main destroying form of rails, so the PD3 rail with high mechanical intension should be selected. But in the high-speed route, when the fatigue is the main factor, the rail of U71Mn should be selected. And for the area that the fatigue easily occurs, especially in the curve sect of rail, the rail polishing technology should be adopted to prevent the cracks in the generating stage of crack.

\section{References}

A. Bernasconi, P. Davoli, M. Filippini \& S. Foletti. (2005). An integrated approach to rolling contact sub-surface fatigue assessment of railway wheels. Wear. No.258. P.973-980.

Anders Ekberg \& Elena Kabo. (2005). Fatigue of railway wheels and rails under rolling contact and thermal loading-an overview. Wear. No.258. P.1288-1300.

D W Barke \& W K Chiu. (2005). A review of the effects of out-of-round wheels on track and vehicle components. Journal of Rail and Rapid Transit. No.219. P.151-175.

Jin, Xuesong \& Shen, Zhiyun. (2001). New Developments of the Rolling Contact Fatigue of Wheel Track. Journal of the China Railway Society. No.23(2). P.92-108.

Jonas W. Ringsberg. (2001). Life prediction of rolling contact fatigue crack initiation. International Journal of Fatigue. No.23. P.575-586.

Liu, Jianfeng \& Wei, Qingchao. (2000). Prediction of Fatigue Service Life for Rails of High-speed Rialway. Journal of Railway Engineering Society. No.2. P.30-34.

Liu, Qiyue, Wang, Xiaqiu \& Zhou, Zhongrong. (1998). An Investigation on Rail Corrugation. Tribology. No.18(4). P.337-340. 
Liu, Qiyue, Zhang, Bo \& Zhou, Zhongrong. (2003). Experimental Study on Rolling Wheel Corrugation. Tribology. No.23(2). P.132-135.

Liu, Qiyue, Zhang, Bo \& Zhou, Zhongrong. (2002). The Research of Wear Characteristics on Steel Rail. China Mechanical Engineering. No.13(18). P.1596-1599.

Lu, Zuwen. (2007). Overall Comments on Track Technology of High-speed Railway. Journal of Railway Engineering Society. No.1. P.41-54.

Wang, Wenjian \& Liu, Qiyue. (2007). Study on Fatigue Crack Growth Rate of PD3 and U71Mn Rail Steel. Journal of Mechanical Strength. No.29(6). P.1026-1029.

Zhao, Xueqin. (2007). Study on the Forming Mechanism of Rail Contact Fatigue Cracks. Chengdu: Master's degree thesis of Southwest Jiaotong University.

Table 1. Chemical components and contents of two kinds of rail steel

\begin{tabular}{|c|c|c|c|c|c|}
\hline Rail steel & $\mathrm{C} \%$ & $\mathrm{Si} \%$ & $\mathrm{Mn} \%$ & $\mathrm{P} \%$ & $\mathrm{~S} \%$ \\
\hline U71Mn hot rolled & 0.736 & 0.282 & 1.4 & 0.02 & 0.033 \\
\hline PD3 hot rolled & 0.793 & 0.712 & 0.771 & 0.022 & 0.014 \\
\hline
\end{tabular}

Table 2. Mechanical performances of two kinds of rail steel under normal temperature

\begin{tabular}{|c|c|c|c|}
\hline Rail steel & $\mathrm{d} 5(\%)$ & $\sigma 0.2(\mathrm{Mpa})$ & $\sigma \mathrm{b}(\mathrm{Mpa})$ \\
\hline U71Mn hot rolled & 10 & 644.5 & 1077.5 \\
\hline PD3 hot rolled & 10 & 552 & 964 \\
\hline
\end{tabular}

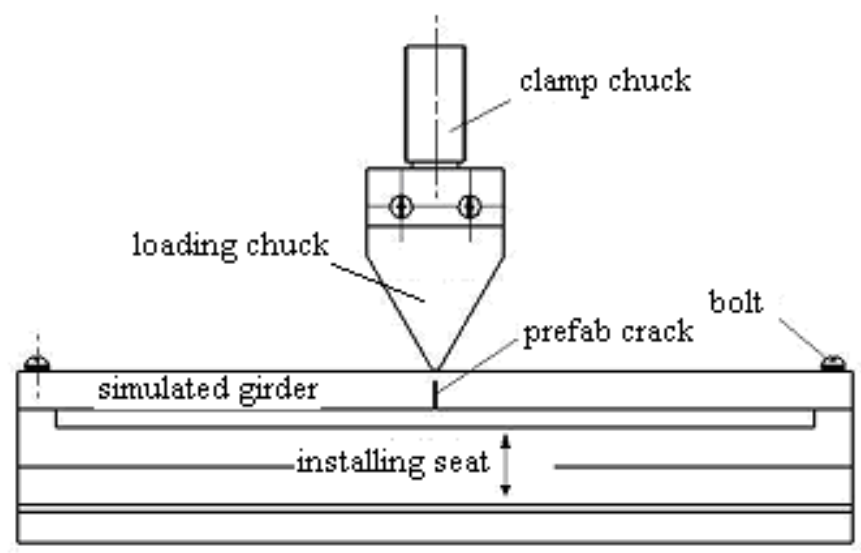

Figure 1. Sketch Map of Experiment Structure (Unit: mm)

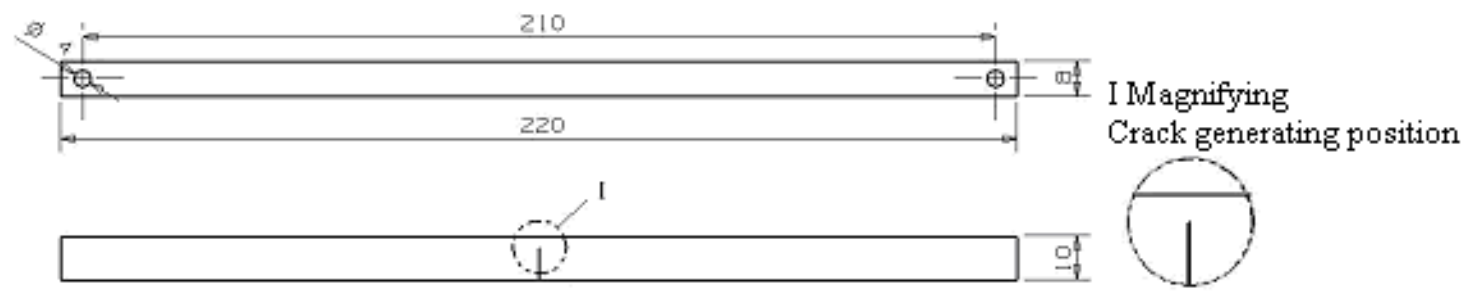

Figure 2. Sketch Map of Simulated Girder Size and Crack Generating Position (Unit: mm) 


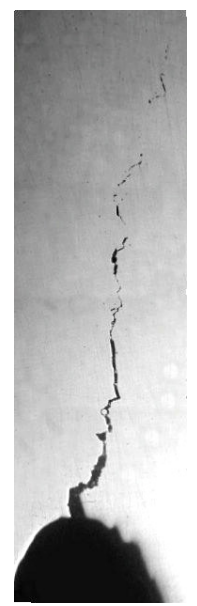

Figure 3. U71Mn Static Load Crack

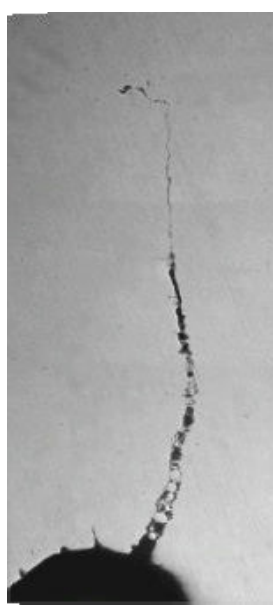

Figure 5. U71Mn Crack after 12000 Times Fatigue

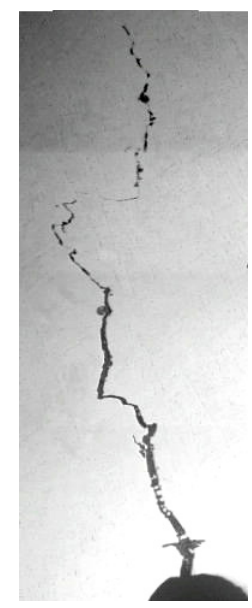

Figure 4. PD3 Static Load Crack

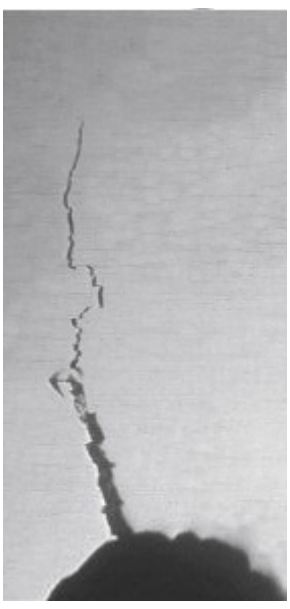

Figure 6. PD3 Crack after 4000 Times Fatigue

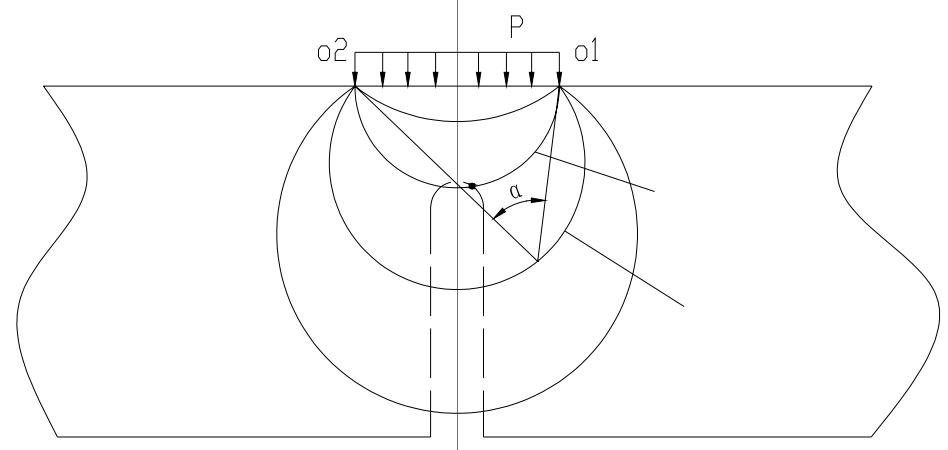

Figure 7. Simple Analysis of Main Shearing Stress of Simulated Girder 


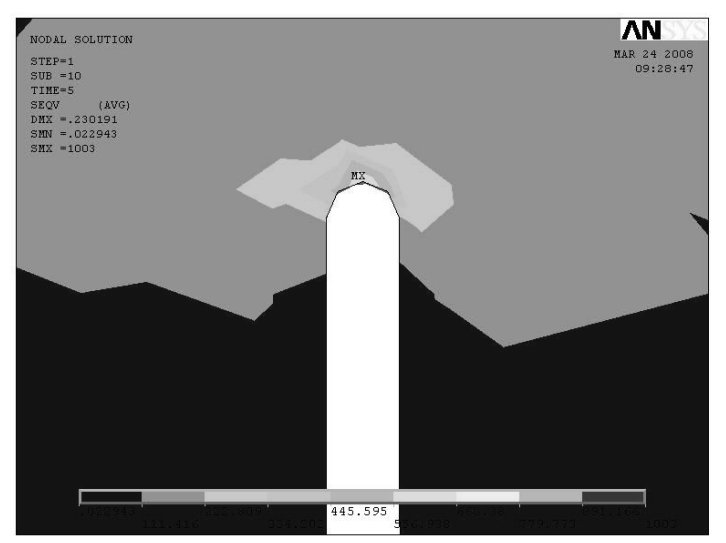

Figure 8. Equivalent Stress Distribution of Crack Top

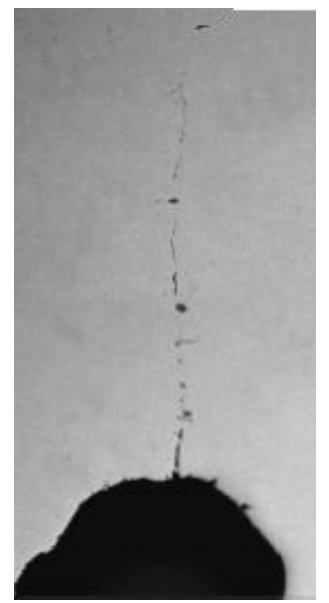

Figure 9. U71Mn Rail Steel Crack after 21000 Times Fatigue 